\title{
Lung cancer risk in a population-based cohort of patients hospitalized for asthma in Sweden
}

\author{
P. Boffetta*,\#, W. Ye" ${ }^{\#}$, G. Boman $₫$, O. Nyrén"
}

Lung cancer risk in a population-based cohort of patients hospitalized for asthma in Sweden. P. Boffetta, W. Ye, G. Boman, O. Nyrén. C) ERS Journals Ltd 2002.

ABSTRACT: It has been suggested that asthma increases the risk of lung cancer in males but not in females. However, previous studies may suffer from report bias and are based on a small numbers of cases.

The objective of the present study was to assess the incidence of lung cancer in males and females using a nationwide Swedish cohort of asthma patients.

Patients $(\mathrm{n}=\mathbf{9 2 , 9 8 6 )}$ aged $\geqslant 20$ yrs with a hospital-discharge diagnosis of asthma and who were alive and free from malignancy $1 \mathrm{yr}$ after first hospitalization were followedup, for incidence of lung cancer during the period 1965-1994 (average duration of follow-up, $8.5 \mathrm{yrs}$ ). Their incidence of lung cancer was compared with that of the national population.

The authors observed 713 lung cancers (standardized incidence ratio (SIR) 1.58, 95\% confidence interval (CI) 1.47-1.70). The SIR was 1.51 in males $(95 \%$ CI $1.38-1.65,492$ cases) and 1.78 in females $(95 \%$ CI $1.55-2.03,221$ cases $)$. The SIR decreased with duration of follow-up and increased with calendar period and age at first hospitalization. The risk of lung cancer was higher for squamous cell and small cell carcinoma than for adenocarcinoma, and it was higher in patients with other diseases as the main diagnosis and in patients hospitalized in departments other than internal and respiratory medicine.

It was confirmed that asthma patients are at increased risk of lung cancer, but there is no heterogeneity in risk between the sexes. Several indirect arguments point towards a noncausal explanation of these findings; in particular, confounding by tobacco smoking is a plausible explanation.

Eur Respir J 2002; 19: 127-133.
*Unit of Environmental Cancer Epidemiology, International Agency for Research on Cancer, Lyon, France. ${ }^{\#}$ Dept of Medical Epidemiology, Karolinska Institute, Stockholm, and Dept of Medical Sciences, Respiratory Medicine and Allergology, Uppsala University, Uppsala, Sweden.

Correspondence: P. Boffetta

International Agency for Research on Cancer

150 cours Albert-Thomas

69008 Lyon

France

Fax: 33472738320

Keywords: Asthma

epidemiology

lung neoplasms

Received: May 172001

Accepted after revision October 13 2001
An imbalance between generation and elimination of oxygen $\left(\mathrm{O}_{2}\right)$ and xenobiotic derived free radicals has been implicated in the aetiology and perpetuation of asthma [1]. The chronic bronchiolar inflammation, with activation of neutrophils and phagocytes, generates, in itself, reactive $\mathrm{O}_{2}$ species [2] and there is evidence that the antioxidant levels in the respiratory tract lining of asthmatic patients are reduced [3]. Free radicals have the potential of causing genetic damage, and a local abundance may therefore contribute to malignant transformation. The epidemiological literature points towards an association between previous history of asthma and lung cancer [4-9]. However, some studies have reported no association [10-12], in particular among females [5, $6,13-15]$. The difference between sex, if true, might either reflect a different susceptibility or result from interaction with other factors, such as smoking (two of the studies with null results in females were conducted among nonsmokers $[15,16])$ and occupational exposures. One problem in the interpretation of the available evidence derives from the fact that most available studies are retrospective, and therefore susceptible to selection and recall bias. Moreover, lack of validity of self-reports of asthma [17], may contribute to nondifferential misclassification of exposure, resulting in attenuation of risk estimates.

Prospective population-based studies based on hospital-discharge records overcome these limitations, since they represent the experience of an unselected series of patients and asthma is assessed before the diagnosis of lung cancer. In addition, classification of exposure relies on hospital diagnoses rather than self reports. To the best of the authors' knowledge, only one small prospective study has been reported, from Finland, that suggested an increased risk of lung cancer among 471 asthmatic patients [8]. The results of the follow-up of a large series of patients hospitalized for asthma, in Sweden, during 1965-1994 are reported in this study. The authors specifically aimed at testing the hypothesis that asthma (or its treatment) increases the risk of lung cancer in males but not in females. In addition, the risk of lung cancer according to other characteristics of asthma patients, such as age, and the risk of specific histological types of lung cancer was assessed.

\section{Methods}

Since 1964, the Swedish National Board of Health and Welfare has collected computerized information 
on individual hospital discharges in the Inpatient Register. The coverage of the population has increased steadily: in 1969 , the register covered $60 \%$ of the population of the country and in 1987 it attained nationwide coverage. Each record of the register includes the national registration number (a unique personal identifier assigned to all Swedish residents), the dates of admission and discharge, up to six discharge diagnoses, and data on hospital departments. During the period covered by this investigation, the discharge diagnoses were coded according to modified versions of the seventh revision of the International Classification of Diseases (ICD-7) until 1968 [18], of the eighth revision (ICD-8) until 1986 [19], and of the ninth revision (ICD-9) thereafter [20].

All records in the Inpatient Register, containing a hospital-discharge diagnosis of asthma (modified ICD-7 codes 241.00 and 241.01, modified ICD-8 codes 493.00 and 493.09, modified ICD-9 codes 493A, 493B and $493 \mathrm{X}$ ), at age $\geqslant 20$ yrs during 1965-1994 were considered. The diagnosis of allergic rhinitis combined with asthma was excluded. The authors identified 118,106 unique national registration numbers with at least one such record. The national registration numbers were linked to the nationwide registries of the total population, causes of death and emigration, to identify records with incorrect national registration numbers. This linkage resulted in the exclusion of 4,243 records that did not match with any of these registries. The linkage with the mortality and emigration registries also provided the dates of death or emigration for some persons, and it resulted in the exclusion of 3,017 patients who died during the hospitalization. Finally the cohort was linked to the national Cancer Registry, founded in 1958 and estimated to be $98 \%$ complete [21], in order to identify cases of cancer that occurred among the patients in the cohort, either before (prevalent cases) or after (incidence cases) the first hospital discharge with diagnosis of asthma. Cancers were classified according to the ICD-7 [22]. Since analysis was restricted to incidence cancers only, 8,157 patients with a prevalent cancer were excluded. A further 161 patients were excluded at various steps of the linkage procedures because of inconsistencies of sex, date of birth or death, etc. A total of 102,528 patients with valid data were retained. The first year of observation following the first hospitalization was excluded, in order to reduce the selection bias that could occur because asthma patients with a yet undetected subclinical cancer are more likely to be hospitalized than other asthma patients. This resulted in the exclusion of 9,542 patients who developed a cancer, died or emigrated within $1 \mathrm{yr}$ of the first hospital discharge, or who were followed-up for $<1 \mathrm{yr}$. The final cohort of patients with valid data, who were alive and free from cancer 1 yr after first hospitalization, comprised 92,986 individuals.

The cohort members were thus followed-up from the first day of the second year following discharge after the first recorded (index) hospitalization for asthma until emigration, occurrence of cancer, death, or end of follow-up (December 31, 1995), whichever occurred first. The observed cancer events in the analysis were the first cancers diagnosed in cohort members, excluding latent cancers incidentally detected at autopsy.

The standardized incidence ratio (SIR), defined as the ratio of the observed to the expected cases of cancer, as the measure of association was used. The expected number of cases was calculated by multiplying Swedish sex, 5-yr age group and calendar yearspecific cancer incidence rates by the observed number of person-yrs in each stratum. The incidence rates used in this calculation were derived from the entire Swedish population without reported cancer, excluding cancers first detected at autopsy. The 95\% confidence interval (CI) for each SIR was calculated by the exact method on the assumption of a Poisson distribution of observed cases.

In separate analyses, person-yrs of observation were divided according to time elapsed since the index hospitalization. No information was available on severity or duration of asthma, nor on treatment received by patients. However, information on other discharge diagnoses at the time of the index admission was available and the cohort could be stratified according to whether asthma was the only, the primary or a secondary diagnosis. Similarly, information on other diseases at the index or subsequent admissions of the same patients allowed for the separation of patients who had at any time been hospitalized for emphysema or chronic bronchitis. Additional analyses were based on stratification on number of hospitalizations for asthma during the first year of follow-up $(1,2-3, \geqslant 4)$ and on department of index hospitalization (internal medicine or respiratory medicine, other departments). In the case of quantitative or semiquantitative variables, the monotonic increasing or decreasing trends in SIRs were tested by using a Chi-squared test [23].

Analyses by histological type of lung cancer were also conducted: squamous cell carcinoma, adenocarcinoma and small cell carcinoma. For the latter type, the analysis was restricted to the period 1987-1994, because the corresponding reference rates were not available for the earlier period.

In order to control simultaneously for several variables, multiplicative Poisson regression models were fitted, using the expected number of cases in each stratum as offset. The regression approach generated relative risks (RRs) of lung cancer and their 95\% CIs. The fit of the regression models were checked using Pearson's Chi-squared statistic. For testing the trend of RRs, the difference of deviances derived from the models, including and excluding the explanatory variable treated semicontinuously, to be interpreted as a Chi-squared statistic with one degree of freedom was calculated [23]. All analyses were performed using the SAS statistical package; in particular, the PROC GENMOD program to fit Poisson regression models was used.

\section{Results}

Table 1 provides summary information on the cohort used in the study. The patients in the cohort 
Table 1.-Selected characteristics of patients with a hospital-discharge diagnosis of asthma in Sweden during 1965-1994

\begin{tabular}{|c|c|c|}
\hline Characteristic & Patients & Person-yrs \\
\hline Total & 92986 & 698688 \\
\hline \multicolumn{3}{|l|}{ Sex } \\
\hline Male & 42663 & 310289 \\
\hline Female & 50323 & 388399 \\
\hline \multicolumn{3}{|l|}{ Duration of follow-up } \\
\hline $1-4 \mathrm{yrs}$ & 35703 & 293741 \\
\hline $5-9$ yrs & 26503 & 216267 \\
\hline$\geqslant 10 \mathrm{yrs}$ & 30780 & 188680 \\
\hline \multicolumn{3}{|l|}{ Calendar year at entry } \\
\hline 1965-1974 & 16036 & 215828 \\
\hline 1975-1984 & 31225 & 299116 \\
\hline 1985-1994 & 45725 & 183743 \\
\hline \multicolumn{3}{|l|}{ Age at entry } \\
\hline $20-44$ & 19711 & 209103 \\
\hline $45-59$ & 22277 & 215411 \\
\hline$\geqslant 60$ & 50998 & 274173 \\
\hline \multicolumn{3}{|l|}{$\begin{array}{l}\text { Presence of other diagnoses } \\
\text { at index hospitalization }\end{array}$} \\
\hline Asthma as only diagnosis & 41129 & 370313 \\
\hline $\begin{array}{l}\text { Other diagnoses, asthma } \\
\text { as main diagnosis }\end{array}$ & 20565 & 157464 \\
\hline $\begin{array}{l}\text { Other diagnoses, asthma } \\
\text { as secondary diagnosis }\end{array}$ & 31292 & 170911 \\
\hline \multicolumn{3}{|l|}{ Dept of index hospitalization } \\
\hline $\begin{array}{l}\text { Internal and } \\
\text { respiratory medicine }\end{array}$ & 74114 & 585231 \\
\hline Other dept & 18872 & 113457 \\
\hline \multicolumn{3}{|l|}{$\begin{array}{l}\text { Number of hospitalization } \\
\text { during first year of follow-up }\end{array}$} \\
\hline 1 & 62638 & 466210 \\
\hline $2-3$ & 22872 & 176998 \\
\hline$\geqslant 4$ & 7476 & 55480 \\
\hline \multicolumn{3}{|l|}{ Presence of emphysema ${ }^{\#}$} \\
\hline No & 91593 & 691788 \\
\hline Yes & 1393 & 6899 \\
\hline \multicolumn{3}{|l|}{ Presence of chronic bronchitis ${ }^{\#}$} \\
\hline No & 73679 & 600372 \\
\hline Yes & 19307 & 98315 \\
\hline
\end{tabular}

\#: person-yrs before the first hospitalization for emphysema or chronic bronchitis were allocated to the stratum without the disease.

provided 698,688 person-yrs of observation (mean duration of follow-up, $8.5 \mathrm{yrs}$, including the first year after index hospitalization, which did not contribute person-yrs of observation). Males comprised $45.9 \%$ of the cohort and contributed $44.4 \%$ of person-yrs: they experienced on average a shorter follow-up $(8.3 \mathrm{yrs})$ than females $(8.7$ yrs). The mean age at first hospitalization was $59.1 \mathrm{yrs}$, and the mean year of first hospitalization was 1983 . The increasing number of patients enrolled in the cohort with duration of follow-up is explained by the increasing coverage of the Inpatient Registry.

The number of observed cases of lung cancer was in excess of the expected number, resulting in a SIR of $1.58(95 \%$ CI 1.47-1.70) (table 2). The SIR was increased in both females (SIR 1.78, 95\% CI 1.55-2.03) and males (SIR 1.51, 95\% CI 1.38-1.65) ( $\mathrm{p}$-value of difference between sex was 0.04). In both sexes, the increase in SIR was restricted to the first
9 yrs of follow-up and was less marked in cohort members who had their index hospitalization before 1975. An effect of age at index hospitalization was present in both sexes, with no increase in SIR in the group of patients with first hospitalization before the age of 45 yrs (it should be noted however that the number of expected cases in this group of patients was small).

The SIR of lung cancer was higher among patients with other diagnoses in addition to asthma, in particular for those in whom asthma was not the main reason for hospitalization, rather than among patients with a diagnosis of asthma only. In this latter group, the increase in SIR of lung cancer was statistically significant during the first 5 yrs of follow-up only, and no increase in risk was apparent after $\geqslant 10$ yrs of follow-up (fig. 1).

The SIR of squamous cell carcinoma and small cell carcinoma was elevated, while the number of cases of adenocarcinoma was close to that expected (table 2). This pattern was present in both sexes.

Since age, calendar period and duration of followup are correlated, the authors aimed at establishing the contribution of each factor through stratified analyses. Figure 2 presents the results stratified by age and calendar year at index hospitalization. Only a modest calendar-period effect was found, and it was apparent in the older patients only, while the effect of age was apparent across the whole study period. In further analyses by age at first hospitalization and duration of follow-up, the difference in SIR by age at first hospitalization was restricted to the first $5 \mathrm{yrs}$ of follow-up (data not shown in detail). A further analysis of duration of follow-up and calendar period provided no further insight into the results; both the modest effect of calendar period and the strong effect of duration of follow-up were confirmed (not shown in detail).

Stratification of the cohort according to presence of emphysema does not suggest confounding by factors associated with the latter disease, although the number of cases with concomitant diagnosis of emphysema was small (table 2). Conversely, a similar stratification according to presence of chronic bronchitis revealed an elevated SIR among patients with asthma and bronchitis (table 2). In particular, the SIR of squamous cell carcinoma among patients with chronic bronchitis was 3.73 (95\% CI 3.11-4.45). A small but significant increase in SIR was however also present among patients without chronic bronchitis (table 2, 1.79 [1.55-2.07] for squamous cell carcinoma).

The SIR of lung cancer was higher among patients with only one hospitalization for asthma during the first year of follow-up than in other patients. The SIR was lower in patients hospitalized in internal or respiratory medicine than in other patients ( $\mathrm{p}$-value of difference $<0.01$; table 2 ). Additional analyses were conducted by attained age, as opposed to age at first hospitalization as shown in table 2 , but there was no indication of a modification of the risk of lung cancer according to this variable (results not shown in detail).

The results of the Poisson regression analysis are presented in table 3 . The results found in the 
Table 2. - Standardized incidence ratios (SIRs) and 95\% confidence intervals (Cls) of lung cancer among asthma patients according to selected characteristics of the cohort

\begin{tabular}{|c|c|c|c|c|c|c|c|c|c|}
\hline & \multicolumn{3}{|c|}{ Males } & \multicolumn{3}{|c|}{ Females } & \multicolumn{3}{|c|}{ Total } \\
\hline & Obs & SIR & $95 \% \mathrm{CI}$ & Obs & SIR & $95 \% \mathrm{CI}$ & Obs & SIR & $95 \% \mathrm{CI}$ \\
\hline Total & 492 & 1.51 & $1.38-1.65$ & 221 & 1.78 & $1.55-2.03$ & 713 & 1.58 & $1.47-1.70$ \\
\hline \multicolumn{10}{|l|}{ Duration of follow-up } \\
\hline $1-4 \mathrm{yrs}$ & 274 & 1.99 & $1.76-2.24$ & 110 & 2.32 & $1.91-2.80$ & 384 & 2.07 & $1.87-2.29$ \\
\hline $5-9$ yrs & 130 & 1.31 & $1.10-1.56$ & 61 & 1.62 & $1.24-2.08$ & 191 & 1.40 & $1.20-1.61$ \\
\hline$\geqslant 10 \mathrm{yrs}$ & 88 & 0.99 & $0.79-1.22$ & 50 & 1.27 & $0.94-1.68$ & 138 & 1.08 & $0.90-1.27$ \\
\hline Test for linear trend $\mathrm{d}^{\#}$ & & $<0.01$ & & & $<0.01$ & & & $<0.01$ & \\
\hline \multicolumn{10}{|l|}{ Calendar year at entry } \\
\hline $1965-1974$ & 117 & 1.15 & $0.95-1.38$ & 41 & 1.29 & $0.93-1.75$ & 158 & 1.18 & $1.01-1.38$ \\
\hline 1975-1984 & 226 & 1.59 & $1.39-1.81$ & 83 & 1.54 & $1.23-1.91$ & 309 & 1.57 & $1.40-1.76$ \\
\hline $1985-1994$ & 149 & 1.81 & $1.53-2.13$ & 97 & 2.50 & $2.03-3.05$ & 246 & 2.03 & $1.79-2.30$ \\
\hline Test for linear trend ${ }^{\#}$ & & $<0.01$ & & & $<0.01$ & & & $<0.01$ & \\
\hline \multicolumn{10}{|l|}{ Age at entry } \\
\hline $20-44$ & 13 & 0.87 & $0.46-1.49$ & 16 & 1.27 & $0.72-2.06$ & 29 & 1.05 & $0.71-1.51$ \\
\hline $45-59$ & 122 & 1.19 & $0.99-1.43$ & 67 & 1.53 & $1.19-1.94$ & 189 & 1.30 & $1.12-1.49$ \\
\hline$\geqslant 60$ & 357 & 1.71 & $1.54-1.90$ & 138 & 2.03 & $1.70-2.39$ & 495 & 1.79 & $1.63-1.95$ \\
\hline Test for linear trend & & $<0.01$ & & & 0.02 & & & $<0.01$ & \\
\hline \multicolumn{10}{|l|}{$\begin{array}{l}\text { Other diagnoses at } \\
\text { index hospitalization }\end{array}$} \\
\hline Asthma as only diagnosis & 189 & 1.24 & $1.07-1.43$ & 89 & 1.43 & $1.15-1.76$ & 278 & 1.29 & $1.14-1.45$ \\
\hline Asthma as main diagnosis & 128 & 1.62 & $1.35-1.93$ & 53 & 1.76 & $1.32-2.30$ & 181 & 1.66 & $1.43-1.92$ \\
\hline Asthma as secondary diagnosis & 175 & 1.86 & $1.59-2.15$ & 79 & 2.47 & $1.95-3.08$ & 254 & 2.01 & $1.77-2.27$ \\
\hline \multicolumn{10}{|l|}{ Dept of index hospitalization } \\
\hline Internal/respiratory medicine & 392 & 1.41 & $1.27-1.56$ & 177 & 1.67 & $1.43-1.93$ & 569 & 1.48 & $1.36-1.61$ \\
\hline Other & 100 & 2.09 & $1.70-2.55$ & 44 & 2.42 & $1.76-3.25$ & 144 & 2.18 & $1.84-2.57$ \\
\hline \multicolumn{10}{|l|}{$\begin{array}{l}\text { Number of hospitalizations } \\
\text { during first year of follow-up }\end{array}$} \\
\hline 1 & 348 & 1.66 & $1.49-1.85$ & 149 & 1.81 & $1.53-2.12$ & 497 & 1.70 & $1.56-1.86$ \\
\hline $2-3$ & 110 & 1.30 & $1.06-1.56$ & 55 & 1.70 & $1.28-2.21$ & 166 & 1.41 & $1.21-1.65$ \\
\hline$\geqslant 4$ & 33 & 1.04 & $0.72-1.46$ & 17 & 1.77 & $1.03-2.83$ & 50 & 1.21 & $0.90-1.59$ \\
\hline Test for linear trend & & $<0.01$ & & & 0.78 & & & $<0.01$ & \\
\hline \multicolumn{10}{|l|}{ Presence of emphysema } \\
\hline No & 486 & 1.51 & $1.38-1.65$ & 219 & 1.78 & $1.55-2.03$ & 705 & 1.59 & $1.47-1.71$ \\
\hline \multirow{2}{*}{\multicolumn{10}{|c|}{ Presence of chronic bronchitis }} \\
\hline & & & & & & & & & \\
\hline No & 301 & 1.20 & $1.07-1.34$ & 150 & 1.42 & $1.20-1.67$ & 451 & 1.27 & $1.15-1.39$ \\
\hline Yes & 191 & 2.54 & $2.19-2.93$ & 71 & 3.76 & $2.93-4.74$ & 262 & 2.79 & $2.46-3.15$ \\
\hline \multicolumn{10}{|l|}{ Histological type of lung cancer } \\
\hline Squamous cell carcinoma & 233 & 1.83 & $1.61-2.09$ & 81 & 3.22 & $2.56-4.00$ & 314 & 2.06 & $1.84-2.30$ \\
\hline Adenocarcinoma & 66 & 1.11 & $0.86-1.41$ & 43 & 0.99 & $0.71-1.33$ & 109 & 1.06 & $0.87-1.27$ \\
\hline Small cell carcinoma* & 17 & 1.90 & $1.11-3.05$ & 15 & 3.18 & $1.78-5.25$ & 32 & 2.34 & $1.60-3.31$ \\
\hline
\end{tabular}

Obs: observed. *: p-value; *: follow-up 1987-1994.

univariate analysis were confirmed by the multivariate approach, which suggested only a small reciprocal confounding effect of the factors considered in the analysis.

\section{Discussion}

The study overcomes many of the limitations of previous reports on the risk of lung cancer among asthmatic patients. It is based on prospectively collected information from medical records and includes a large group of cases of lung cancer. The analysis confirms the notion that asthma patients are at increased risk of lung cancer. Contrary to some previous observations, the risk is of comparable magnitude in males and females. Given the large study size, chance can hardly be invoked as an explanation of the results. The prospective nature of the investigation, the reliance on medical diagnoses rather than self reports, and the high quality of the sources of information used to assess hospitalization for both asthma and lung cancer argue against a spurious result due to bias in the selection of patients or in the assessment of information on either asthma or lung cancer.

It should be noted that the exclusion of the first year after index hospitalization might have contributed to an underestimation of the observed number of cases of lung cancer. In fact, if diagnostic bias has operated (i.e. if diagnosis of lung cancer might have been anticipated because of diagnostic or other medical procedures connected with asthma hospitalization), cases of lung cancer which would have occurred during the follow-up might have been concentrated in the first year after hospitalization 


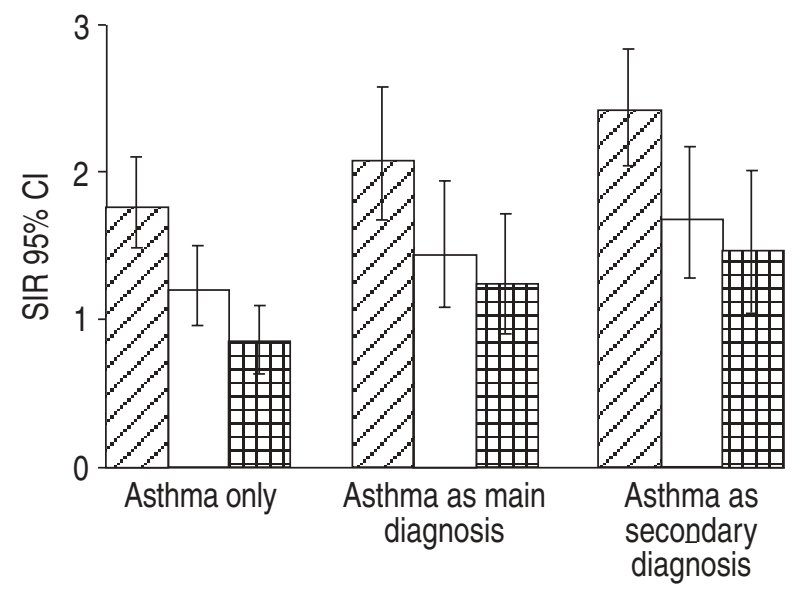

Fig. 1.-Standardized incidence ratio (SIR) of lung cancer among patients hospitalized for asthma, by years of follow-up ( $\Delta$ :

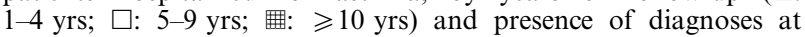
index hospitalization. CI: confidence interval.

(and therefore excluded from the present study). Given the short latent period of lung cancer, however, it is unlikely that such bias might have been of great magnitude. As mentioned earlier, the exclusion of the first year of follow-up was justified by the need to reduce selection bias.

If chance and bias can be reasonably excluded, three explanations can be proposed for the observed

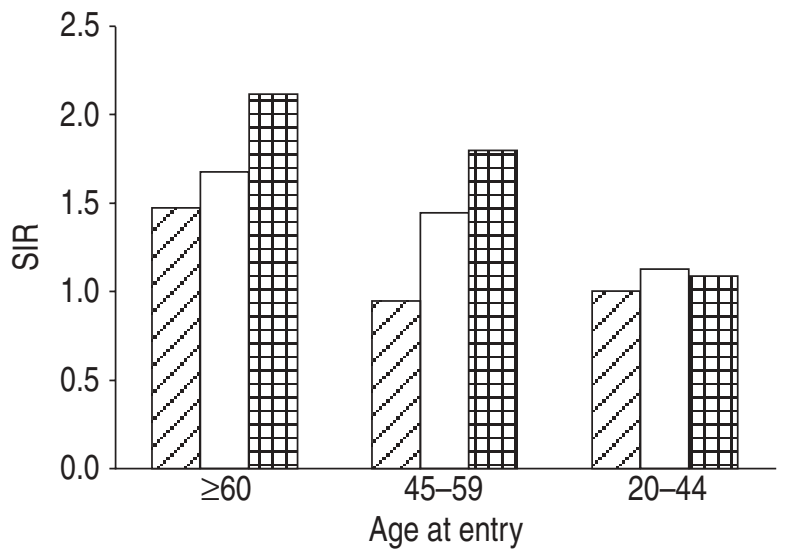

Fig. 2.-Standardized incidence ratio (SIR) of lung cancer among patients hospitalized for asthma, by age at entry and calendar period at entry ( $\square$ : 1965-1974; $\square$ : 1975-1984; 曲: 1985-1994).

association. First, the association is due to confounding by a factor common to asthma and lung cancer. Second, a diagnosis of asthma is made on the basis of early symptoms of lung cancer. Third, either asthma represents a causal or contributing factor in lung carcinogenesis or the two diseases share some mechanistic event.

Although the data needed to fully address these three alternatives was lacking, several elements of the analysis may provide indirect arguments. Tobacco smoking is a candidate for confounding. The role of

Table 3.-Results of the multivariate analyses, according to selected cohort characteristics ${ }^{f}$

\begin{tabular}{|c|c|c|c|c|c|c|}
\hline & \multicolumn{2}{|c|}{ All cancers } & \multicolumn{2}{|c|}{ Adenocarcinoma } & \multicolumn{2}{|c|}{ Squamous cell carcinoma } \\
\hline & $\mathrm{RR}^{\#}$ & $95 \% \mathrm{CI}^{\oplus}$ & $\mathrm{RR}^{\#}$ & $95 \% \mathrm{CI}^{\top}$ & $\mathrm{RR}^{\#}$ & $95 \% \mathrm{CI}^{\oplus}$ \\
\hline \multicolumn{7}{|l|}{ Duration of follow-up } \\
\hline $1-4 \mathrm{yrs}^{+}$ & 1.0 & & 1.0 & & 1.0 & \\
\hline $5-9$ yrs & 0.69 & $0.58-0.82$ & 0.48 & $0.29-0.78$ & 0.72 & $0.56-0.93$ \\
\hline$\geqslant 10 \mathrm{yrs}$ & 0.55 & $0.45-0.67$ & 0.56 & $0.35-0.90$ & 0.49 & $0.36-0.67$ \\
\hline Test fore linear trend $d^{\S}$ & $<0.01$ & & $<0.01$ & & $<0.01$ & \\
\hline \multicolumn{7}{|l|}{ Dept at first hospitalization } \\
\hline Internal/respiratory medicine ${ }^{+}$ & 1.0 & & 1.0 & & 1.0 & \\
\hline Other & 1.31 & $1.08-1.58$ & 1.38 & $0.84-2.27$ & 1.32 & $0.99-1.76$ \\
\hline \multicolumn{7}{|l|}{$\begin{array}{l}\text { Number of hospitalizations } \\
\text { during first year of follow-up }\end{array}$} \\
\hline $1^{+}$ & 1.0 & & 1.0 & & 1.0 & \\
\hline $2-3$ & 0.83 & $0.70-0.99$ & 0.92 & $0.58-1.44$ & 0.97 & $0.75-1.25$ \\
\hline$\geqslant 4$ & 0.71 & $0.53-0.95$ & 1.03 & $0.52-2.02$ & 0.78 & $0.51-1.20$ \\
\hline Test for linear trend ${ }^{\S}$ & $<0.01$ & & 0.90 & & & \\
\hline \multicolumn{7}{|l|}{ Presence of emphysema } \\
\hline $\mathrm{No}^{+}$ & 1.0 & & 1.0 & & 1.0 & \\
\hline Yes & 0.72 & $0.36-1.45$ & 2.02 & $0.64-6.40$ & 0.58 & $0.19-1.81$ \\
\hline \multicolumn{7}{|l|}{ Presence of chronic bronchitis } \\
\hline $\mathrm{No}^{+}$ & 1.0 & & 1.0 & & 1.0 & \\
\hline Yes & 2.17 & $1.86-2.54$ & 2.30 & $1.54-3.43$ & 2.28 & $1.81-2.87$ \\
\hline \multicolumn{7}{|l|}{$\begin{array}{l}\text { Other diagnoses at } \\
\text { index hospitalization }\end{array}$} \\
\hline Asthma as only diagnosis ${ }^{+}$ & 1.0 & & 1.0 & & 1.0 & \\
\hline Asthma as main diagnosis & 1.10 & $0.91-1.33$ & 0.82 & $0.49-1.37$ & 1.05 & $0.79-1.41$ \\
\hline Asthma as secondary diagnosis & 1.20 & $1.00-1.43$ & 1.12 & $0.71-1.75$ & 1.30 & $1.00-1.70$ \\
\hline
\end{tabular}

$f_{:}$the estimated standardized incidence ratio for patients with all the reference characteristics listed in the table were 1.55 (all lung cancers), 1.12 (adenocarcinoma) and 1.84 (squamous cell carcinoma); \#: relative risk (RR) adjusted mutually for variables listed in table $2{ }^{\uparrow}$ : confidence interval $(\mathrm{CI}){ }^{+}$: reference category; ${ }^{\S}$ : p-valve. 
tobacco smoking in the causation of atopic asthma has not been demonstrated [24, 25]; however, smoking may exacerbate the clinical course of the disease [26]. Furthermore, it is possible that other chronic pulmonary diseases, likely to be associated with tobacco smoking, might have been misdiagnosed as asthma in patients in this study, as well as in other studies [27]. In conclusion, it is plausible that the asthma patients included in the present study smoked more than the general Swedish population, as has been found in other studies [28].

No information on the individual smoking habits of the patients included in the study was available. However, several indirect arguments are consistent with a confounding effect exerted by tobacco smoking. The SIR was higher for squamous and small cell carcinoma than for adenocarcinoma, paralleling the strength of the carcinogenic role of tobacco on different histological types of lung cancer. The decrease in risk of lung cancer following first hospitalization for asthma (which is consistent with the only other similar results reported in the literature [8]) might reflect the beneficial effect of quitting smoking (asthma patients have a higher proportion of former smokers than healthy individuals [24]). In the only study presenting lung cancer risk estimates with and without adjustment for tobacco smoking, a confounding effect was suggested [8]. In conclusion, the authors were not able to fully assess the possible contribution of other causes of lung cancer, and notably tobacco smoking, on the observed association; confounding by tobacco smoking, however, remains a plausible explanation.

An argument in favour of reverse causality (i.e. a diagnosis of asthma made following the early symptoms to the early neoplastic process or the inflammation associated with it) is the clear decrease in SIR of lung cancer with duration of follow-up. The presence of an increased SIR in the group of patients with 5-9 yrs of follow-up, however, cautions against a straightforward interpretation along these lines, given the relatively rapid development of bronchial and lung neoplasms. As discussed earlier, the decrease in SIR with duration of follow-up can also be interpreted as an effect of decrease in tobacco consumption following the first hospitalization for asthma.

Although the hypothesis of a causal interpretation is supported by the clear increase in risk in both sexes, several aspects of the results, in addition to those discussed earlier, point towards a noncausal interpretation. The risk of lung cancer was higher among patients with other diseases concomitant with asthma than among patients with asthma as the only diagnosis. In particular the risk was highest among those patients with asthma as a secondary rather than a primary diagnosis. The strong gradient in risk according to age at index hospitalization is also an argument against causality, since older individuals are less likely to represent a pure population of patients with asthma than younger patients. It is noteworthy that the SIR after 10 yrs of follow-up among patients aged $<45$ yrs at index hospitalization is 1.00 (19 observed cases). Finally, diagnosis of asthma is likely to have been of higher specificity in departments of internal and respiratory medicine, while the risk of lung cancer was higher in patients hospitalized in other departments.

A modest calendar-period effect (i.e. patients who had their first hospitalization after 1975 had a higher risk of lung cancer than other patients) was found in the present study. This finding might suggest that temporal changes in the management of asthma may have contributed to the increase in lung-cancer risk. However, the interpretation of the results on calendar period is complicated by the lack of a complete history among patients whose first hospitalization occurred early in the study period, since they are more likely than other patients to have been hospitalized before the beginning of the period of observation of the study.

The study confirms the presence of an association between asthma and lung cancer; contrary to some previous reports, no difference in risk was found between sexes. This higher risk in males as compared to females observed in earlier studies, mainly of the retrospective design, might be due to differences in the effect of confounding by smoking between the two sexes or in the bias from the self reporting of previous medical conditions. A plausible explanation of the results is that the association observed in this study (as well as in previous investigations) is due to confounding by tobacco smoking. The confounding effect may be due to a role of tobacco smoking in chronic pulmonary conditions that were misclassified as asthma rather than in asthma itself.

An additional potential confounder are occupational exposures that are associated with both asthma and lung cancer. It is likely, however, that any confounding effect of occupation would be small in comparison with that of tobacco smoking. A causal interpretation of the association observed in the present study, however, also remains plausible. It would indicate an effect of asthma-associated factors relevant to lung carcinogenesis. These may include generation and elimination of free radicals [1], reduction of antioxidant levels in the respiratory tract [3], as well as chronic bronchiolar inflammation, which would further generate reactive oxygen species [2]. If chronic inflammation is an important factor, it would imply an effect primarily on the late stages of the carcinogenic process of the lung, mainly among patients with asthma diagnosed late in life. The prospective observation of young asthma patients for a longer period of follow-up, than was feasible in this study, would provide important information on the timing of a carcinogenic effect of asthma (if any) on the lung.

\section{References}

1. Tekin D, Sin BA, Mungan D, Misirligil Z, Yavuzer S. The antioxidative defense in asthma. J Asthma 2000; 37: 59-63.

2. Kelly FJ. Gluthatione: in defence of the lung. Food Chem Toxicol 1999; 37: 963-966.

3. Comhair SA, Bhathena PR, Dweik RA, Kavuru M, Erzurum SC. Rapid loss of superoxide dismutase 
activity during antigen-induced asthmatic response. Lancet 2000; 355: 624.

4. Robinette CD, Fraumeni JF Jr. Asthma and subsequent mortality in World War II veterans. $J$ Chron Dis 1978; 31: 619-624.

5. Vena JE, Bona JR, Byers TE, Middleton E Jr, Swanson MK, Graham S. Allergy-related diseases and cancer: an inverse association. Am J Epidemiol 1985; 122: 66-74.

6. Reynolds P, Kaplan GA. Asthma and cancer. Am J Epidemiol 1987; 125: 539-540.

7. Vesterinen E, Pukkala E, Timonen T, Aromaa A. Cancer incidence among 78,000 asthmatic patients. Int J Epidemiol 1993; 22: 976-982.

8. Huovinen E, Kaprio, Vesterinen E, Koskenvuo M. Mortality of adults with asthma: a prospective cohort study. Thorax 1997; 52: 49-54.

9. Mayne ST, Buenoconsejo J, Janerich DT. Previous lung disease and risk of lung cancer among men and women nonsmokers. Am J Epidemiol 1999; 149: 13-20.

10. Alderson M. Mortality from malignant disease in patients with asthma. Lancet 1974; ii: 1475-1477.

11. Markowe HLJ, Bulpitt CJ, Shipley MJ, Rose G, Crombie DL, Fleming DM. Prognosis in adult asthma: a national study. $\mathrm{Br}$ Med J 1987; 295: 949952.

12. Mills PK, Beeson L, Fraser GE, Phillips RL. Allergy and cancer: organ site-specific results from the adventist health study. Am J Epidemiol 1992; 136: 287-295.

13. Wu AH, Yu MC, Thomas DC, Pike MC, Henderson BE. Personal and family history of lung disease as risk factors for adenocarcinoma of the lung. Cancer Res 1988; 48: 7279-7284.

14. Osann KE. Lung cancer in women: the importance of smoking, family history of cancer, and medical history of respiratory disease. Cancer Res 1991; 51: 48934897.

15. Alavanja MCR, Brownson RC, Boice JD Jr, Hock E. Preexisting lung disease and lung cancer among nonsmoking women. Am J Epidemiol 1992; 136: 623-632.

16. Wu AH, Fontham ETH, Reynolds P, et al. Previous lung disease and risk of lung cancer among lifetime nonsmoking women in the United States. $\mathrm{Am}$ J Epidemiol 1995; 141: 1023-1032.

17. Toren K, Brisman J, Jarvholm B. Asthma and asthma-like symptoms in adults assessed by questionnaires: a literature review. Chest 1993; 104: 600-608.

18. NBHW. International statistical classification of diseases, injuries, and causes of death, 1955 revision, Kungl. Medicinalstyrelsen, 1965.

19. NBHW. International statistical classification of diseases, injuries, and causes of death, 1968 revision. Stockholm, National Board of Health and Welfare, 1973.

20. NBHW. International statistical classification of diseases, injuries, and causes of death, 1977 revision. Stockholm, National Board of Health and Welfare, 1986.

21. Mattsson B, Rutqvist LE, Wallgren A. Undernotification of diagnosed cancer cases to the Stockholm Cancer Registry. Int J Epidemiol 1985; 14: 64-69.

22. WHO. International Classification of Diseases, Seventh Revision (ICD-7). Geneva, World Health Organization, 1955.

23. Breslow NE, Day NE. Statistical Methods in Cancer Research, Vol. II, The Design and Analysis of Cohort Studies (IARC Scientific Publications No. 82). Lyon, International Agency for Research on Cancer, 1987.

24. Vesterinen E, Kaprio J, Koskenvuo M. Prospective study of asthma in relation to smoking habits among 14,729 adults. Thorax 1988; 43: 534-539.

25. McWhorter WP, Polis MA, Kaslow RA. Occurrence, predictors, and consequences of adult asthma in NHANESI and follow-up survey. Am Rev Respir Dis 1989; 139: 721-724.

26. Siroux V, Pin I, Oryszczyn MP, Le Moual N, Kauffmann F. Relationships of active smoking to asthma and asthma severity in the EGEA study: epidemiological study on the genetics and environment of asthma. Eur Respir J 2000; 15: 470-477.

27. Jarvholm B, Brisman J, Toren K. The association between epidemiological measures of the occurrence of asthma. Int J Tuberc Lung Dis 1998; 2: 1029-1036.

28. Almind M, Viskum K, Evald T, Dirksen A, KokJensen A. A seven-year follow-up study of 343 adults with bronchial asthma. Dan Med Bull 1992; 39: 561-565. 